\title{
Percées : Traversées des littératures francophones canadiennes d'aujourd'hui
}

\author{
Kathleen KELLETT, Université Ryerson \\ Joëlle PAPILLON, Université McMaster
}

Avec le numéro Percées: Traversées des littératures francophones canadiennes d'aujourd'hui, nous espérons contribuer à l'étude des poétiques et politiques variées des auteurs franco-canadiens et autochtones contemporains. Depuis les travaux fondateurs de François Paré publiés dans les années 1990 et 2000 (nous pensons bien sûr aux Littératures de l'exiguïté en 1992, ainsi qu'aux autres essais qui ont suivi, notamment Théories de la fragilité en 1994 et La distance habitée en 2003), les recherches dans le domaine de la littérature franco-canadienne sont foisonnantes. Ne soulignons que les travaux récents menés ou dirigés par Lucie Hotte ( $L a$ littérature franco-ontarienne depuis 1996, avec François Ouellet, en 2016 ; Introduction à la littérature franco-ontarienne, avec Johanne Melançon, en 2010 ; Habiter la distance, avec Guy Poirier, en 2009), Johanne Melançon (Écrire au féminin au Canada français en 2013), ou encore Benoit Doyon-Gosselin (Pour une herméneutique de l'espace en 2012), et le soutien de maisons

d'édition telles que Prise de Parole, Perce-neige, Nota bene, les Éditions du Blé, ou encore David. Si beaucoup d'attention a, dans un premier temps, été portée aux effets de minorisation dans le corpus franco-canadien, l'institutionnalisation de cette littérature a participé à en déplacer les enjeux.

Le projet de ce numéro est né lors du congrès de la NeMLA (Northeast Modern Language Association) à Toronto au printemps 2015, à l'initiative de Catherine Parayre de l'Université Brock et avec le soutien de Lucie Hotte de l'Université d'Ottawa. Lors de cet événement, trois ateliers portant sur la littérature franco-canadienne avaient rassemblé des chercheur-e-s autour des littératures acadienne, franco-ontarienne et franco-manitobaine : «French-Canadian Writers "hors Québec": Under the Prairie Sky », organisé par Kandace Brill Lombart, chercheure indépendante ; «De parenté à parenté : Destinées acadiennes chez Georgette LeBlanc », organisé par Joëlle Papillon de 1'Université McMaster ; et « La littérature franco-ontarienne et l'institution littéraire », organisé par Kathleen Kellett de l'Université Ryerson. L’intérêt des discussions ayant eu lieu lors de ces ateliers, a fait naître le projet de publication dans la revue Voix plurielles; plusieurs autres chercheur-e-s intéressé-e-s par les littératures francophones canadiennes ont été 
invité-e-s à se joindre au projet et, en fin de compte, les contributrices et contributeur au numéro sont en majorité des collègues qui n’ont pas participé au congrès de la NeMLA en 2015. Nous avons entre autres décidé d'inviter deux chercheures en littérature autochtone - Isabella Huberman et Élise Couture-Grondin - à partager leurs recherches dans ce cadre, par souci de faire un portrait plus complet et surtout plus nuancé des littératures de langue française sur ce territoire.

Dans ce recueil d'articles, nous avons essayé de mieux faire connaître des auteurs de tous les coins de la francophonie canadienne. Tout d'abord, nous mettons en vedette l'auteure acadienne Georgette LeBlanc dont les romans poétiques Alma, Amédé, Prudent et Le grand feu ont attiré l'attention critique par leurs riches interprétations de l'histoire et de la culture acadiennes ainsi que par leur interrogation subtile de la nature du désir. Nous sommes ravies d'ouvrir ce numéro avec un poème inédit de Georgette LeBlanc, «Ici (Take 2) », portant sur son père, Gilles Grégoire LeBlanc. La poète y revient sur la vie et la mort de son père, mettant en relief les attachements complexes qui nous lient à nos proches qui ne « se laiss[ent] point / aimer aisé ». Dans ce bel hommage à l'homme que son père a été, l'on retrouve avec bonheur le souffle poétique inimitable de Georgette LeBlanc. Dans le premier article sur LeBlanc, Julie StLaurent traverse l'œuvre afin de faire ressortir le rôle de l'expérience vécue - incarnée dans un corps sexué - dans son écriture de l'histoire. Les personnages centraux de l'œuvre (Alma, Amédé, Prudent, Cécile) sont témoins de moments significatifs de l'histoire acadienne et cadienne; la mise en scène de leurs voix singulières et de leur expérience charnelle du monde permet à LeBlanc de recentrer 1'«Histoire» sur les individus qui participent à la faire. StLaurent analyse la fonction du sensible, du désir et du plaisir en lien à l'expression de l'expérience humaine dans les quatre romans poétiques de LeBlanc. Évitant la fixation, l'écriture de LeBlanc est toujours mobile, posant le passé en dialogue constant avec le présent. Pour sa part, Joëlle Papillon se concentre sur les deux œuvres les plus récentes de LeBlanc, « $\mathrm{S}(\mathrm{H}) \mathrm{hhhhhhh} »$ et Le grand feu, pour analyser sa représentation des personnages féminins désirants et créateurs. Bien que tout semble les séparer, Cécile et Marguerite ont en commun de cheminer vers l'ouverture au désir ; s'ouvrir aux plaisirs du corps leur permet de trouver leur vrai soi, de fracasser le plafond de verre qui les maintenait dans la conformité à la norme sociale de genre de leur époque. C'est en risquant la mort pour s'incarner dans un corps désirant et sensuel - et par là, libre - que Cécile pourra devenir poète et sortir du récit que les figures paternelles 
avaient créé pour elle. Francis Langevin propose une lecture rapprochée des trois premiers romans poétiques de LeBlanc afin de faire ressortir comment les corps de ses personnages sont entravés et sacrifiés. Selon lui, Alma, Amédé et Prudent renoncent tous à leur quête spatiale individuelle - leur désir de grouiller - afin de privilégier les besoins de leur communauté : Alma s'occupe de ses enfants, Amédé offre les poumons de son accordéon au Village, Prudent s'épuise à motiver les Infortunés pour qu'ils reprennent le bateau en train de les déporter. Langevin interprète cette dynamique sacrificielle dans l'œuvre de LeBlanc sous le signe de l'importance de la transmission pour la constitution d'une collectivité.

Notre traversée de la littérature franco-ontarienne propose des études qui mettent en vedette des auteurs aussi divers qu'Hédi Bouraoui, Paul Savoie, Céline Forcier et Michel Dallaire. Suzanne Crosta offre une lecture nuancée du thème de l'altérité et de l'ouverture envers l'Autre dans le roman La réfugiée de Hédi Bouraoui, auteur franco-ontarien d'origine tunisienne. Dans le contexte d'une interrogation très actuelle sur les difficultés des exilés et des migrants à mener à bien leur recherche d'une société d'accueil hospitalière, ce texte de Bouraoui trace le parcours migratoire d'une réfugiée laotienne dont le nom DorBoa signifie « fleur de lotus ». À partir du concept du «narratoème», créé par Bouraoui pour décrire la fusion du poème narratif et du récit poétique, Crosta se penche sur la polyphonie du texte afin de cerner les modalités de cette quête migratoire qui doit faire face aux politiques discriminatoires imposées aux communautés minoritaires par la société d'accueil. Elle montre la façon dont Bouraoui instaure le dialogue interculturel à travers l'enchaînement des métaphores tout au long du texte qui invite à une collaboration entre soi et l'Autre en une nomadanse où les altérités s'interpellent en un mouvement sans cesse renouvelé. Catherine Parayre explore ensuite la figure de l'écrivain et ses rapports avec la critique et l'institution littéraire dans des écrits satiriques de Paul Savoie et Céline Forcier, deux auteurs qui œuvrent en milieu franco-ontarien minoritaire. En considérant la nouvelle «Comment réussir sa vie» de Savoie ainsi que le roman La Critique de Forcier, Parayre examine les «impostures de l'écriture» chez des écrivailleurs aux aspirations littéraires qui dépassent leurs moyens. La protagoniste de Savoie, Esther Beauchemin, est une chroniqueuse dépourvue de principes journalistiques ; l'écrivain en herbe de Forcier, Pascal Pigeon, va jusqu'à kidnapper une critique qui a osé condamner son livre. Tout en reconnaissant l'aspect ludique de ces textes, Parayre souligne le rôle de l'institution littéraire franco-canadienne minoritaire qui peut manifester une certaine complaisance à l'égard de la 
médiocrité. S'intéressant elle aussi à la question de la représentation de l'écrivain, Johanne Melançon identifie une éthique de la création artistique élaborée par le roman poétique l'anarchie des innocences de l'auteur franco-ontarien Michel Dallaire. En tenant compte de l'influence du poète surréaliste Roland Giguère chez Dallaire, Melançon explore la thématique de la plongée en soi au moyen de l'écriture, le besoin de s'affirmer individuellement tout en allant à la rencontre de l'autre, le désir de se révolter contre les normes sociales. L'écrivain entreprend un voyage intérieur qui l'amène à confronter ses propres personnages insolites tels Gertrude la mouche, Coleslaw le peintre et la dame aux crevettes. Le poète accède à l'inconscient, se métamorphose à travers l'écriture, prend conscience de soi et de l'autre et maintient l'esprit de révolte face à un monde violent.

En étudiant la littérature franco-canadienne, il ne faut pas oublier la mobilité de plusieurs Canadiens qui ont tendance à traverser le pays d'un bout à l'autre sans trop se soucier des frontières. Ainsi faut-il noter que Paul Savoie, que l'on considère franco-ontarien ici, est né à Saint-Boniface au Manitoba. S'agit-il d'un auteur franco-ontarien ou franco-manitobain ? La question est-elle pertinente? Certains textes plus que d'autres exigent une lecture qui tient compte du sentiment d'appartenance à un territoire quelconque. Dans son article pour ce numéro, Kathleen Kellett propose une lecture du roman Le soleil du lac qui se couche de l'écrivain franco-manitobain J.R. Léveillé. Elle y étudie la façon dont Léveillé met en valeur la beauté et la diversité de l'espace urbain, rural et culturel du Manitoba, à partir d'un récit d'amour entre un poète japonais et la narratrice métisse du nom d'Angèle, laquelle aspire à devenir architecte. Selon Kellett, Léveillé utilise la relation amoureuse d'Angèle et d'Ueno pour célébrer la rencontre des altérités et le métissage culturel; cet engouement pour le métissage demeure problématique dans une perspective autochtone, tel que le soulignent Kellett dans cet article et Élise Couture-Grondin dans un article subséquent.

Lorsqu'on parle de territoires et de terres natales, il est important de reconnaître que les peuples indigènes en terre d'Amérique n'ont pas été invités à se prononcer sur les frontières imposées par les pouvoirs coloniaux. Isabella Huberman s'inspire de la notion d'amour décolonial reprise par l'écrivaine et penseuse anishinaabe Leanne Simpson pour analyser Kuessipan. Elle propose que l'auteure innue Naomi Fontaine tisse des nids d'amour décolonial entre les personnages de son roman - notamment entre les mères et les enfants, ainsi qu'entre les Aîné-e-s et les apprenti-e-s de la vie autochtone. Huberman imagine également l'amour 
décolonial comme une relation possible entre l'auteure et son récit - voire entre les lectrices/teurs et l'œuvre - créant des espaces où les rapports coloniaux d'appropriation se trouvent bloqués. Dans l'article en clôture du numéro, Élise Couture-Grondin effectue une lecture antiraciste du livre de correspondances entre allochtones et Autochtones Aimititau! Parlons-nous! Si le projet épistolaire avait pour but de créer un lieu d'amitié littéraire interculturel, Couture-Grondin se penche sur l'oblitération apparente de l'héritage raciste qui affecte pourtant toujours les relations entre les Québécois et les Premières Nations. Elle analyse en particulier deux correspondances très différentes - entre Denise Brassard et Rita Mestokosho ; entre Jean Désy et Nahka Bertrand - afin de faire ressortir la dimension politique de cet « oubli » ou de cette mécompréhension de la race et du racisme de la part des écrivains allochtones.

Bien que les articles réunis dans ce dossier ne partagent pas de thématiques précises, l'on remarque cependant la prépondérance de deux préoccupations : d'une part, la place faite au corps, au désir, à l'amour et à la sensualité ; d'autre part, la place faite à l'autre, entre désir de renouveler le dialogue interculturel et menace d'assimilation. Nous espérons que ces «percées » sauront ouvrir la voie pour d'autres lectures stimulantes d'œuvres francophones d'ici.

Nous voudrions reconnaître ici l'appui de celles qui ont organisé les séances qui ont donné lieu à cette collection d'essais ainsi que l'appui institutionnel reçu de la NeMLA et de nos universités. Merci également à l'équipe éditoriale de la revue Voix plurielles. Enfin, nous sommes immensément reconnaissantes aux nombreux/ses collègues qui ont bien voulu trouver le temps de lire et d'évaluer les articles soumis pour ce numéro. Leurs commentaires et suggestions ont permis aux auteur-e-s de consolider leur réflexion. Qu'elles et ils en soient ici remercié-e-s ! 